\title{
Regional averaged control problems with minimum energy constrained by distributed parabolic systems
}

\author{
Maawiya Ould Sidia,*, Rabie Zine ${ }^{\mathrm{b}}$, Abdel-Baset A. Mohamed ${ }^{\mathrm{c}}$ \\ ${ }^{a}$ RT-M2A Laboratory, Mathematics Department, College of Science, Jouf University, P. O. Box: 2014, Sakaka, Saudi Arabia. \\ ${ }^{b}$ School of Science and Engineering, Al Akhawayn University in Ifrane, Morocco. \\ ${ }^{c}$ Department of Mathematics, College of Science and Humanities in Al-Aflaj, Prince Sattam bin Abdulaziz University, Saudi Arabia.
}

\begin{abstract}
This paper study the regional average controllability problems governed by distributed parabolic systems. We establish the definition and characterization of a system which is regional averaged controllable. The averaged regional control problem with minimum energy is considered and solved using HUM (Hilbert uniqueness method). Thereafter, the case of regional gradient averaged controllability is treated.
\end{abstract}

Keywords: Distributed systems, average controllability, regional controllability, gradient controllability, minimum energy. 2020 MSC: 93B40, 93B52, 49K99, 49J99.

(C)2022 All rights reserved.

\section{Introduction}

Controllability arise in several areas in real-life problems, therefore, several researchers have to work on the development of the methods as well on the establishment of this concept. Following this, there are several works dedicatory to the control and analysis of the parabolic distributed systems, for examples, [2-4]. In the last decades, several researches have been devoted to the study of the problem of regional controllability [8] and also of the gradient controllability [7, 9, 10].

In real-life problems, parameter-dependent system modeling is a difficult processes. In this case of unknown value parameter, it is long process to control each implementation of the system by a single control (using an independent control of the parameter). The average controllability introduced by Zuazua [14], purpose to check the averaged state of a parameterized system instead of the state against the unknown parameter. Moreover, the problem of average controllability has recently been introduced in finite dimensional linear systems [5] and in partial differential equations [6].

In this paper, we discuss the regional averaged optimal control problems constrained by distributed parabolic systems. We use the average parameter dependent $\sigma$ in a real interval $(a, b)$, which brings us back to the idea introduced by Zuazua [14] as a particular case of our problem when $a=0$ and $b=1$.

\footnotetext{
*Corresponding author

Email addresses: msidi@ju.edu.sa (Maawiya Ould Sidi), r.zine@aui.ma (Rabie Zine), abdelbastm@aun.edu.eg (Abdel-Baset A. Mohamed)
}

doi: $10.22436 /$ jmcs.026.04.03

Received: 2021-11-12 Revised: 2021-11-22 Accepted: 2021-11-23 
We begin the paper by giving in the second section a general mathematical formulation and definitions of averaged control problems with minimum energy constrained by distributed parabolic systems. In the third section, we define and solve the problem of regional averaged controllability with minimum energy. The proposed solution characterized using an extend approach of the HUM approach. The fourth section is devoted to the regional gradient averaged control problem with minimum energy.

\section{Statement of the problem}

Let $\Omega$ be an open regular bounded set of $\mathbb{R}^{n}$ with boundary $\partial \Omega$. Consider the state-space system

$$
\begin{cases}\frac{d u}{d t}=A(\sigma) u+B p & \Omega \times(0, T), \\ u=0 & \partial \Omega \times(0, T), \\ u(0)=u_{0} & D(A(\sigma)),\end{cases}
$$

where $A(\sigma)$ is an operator on the state space depending on the uncertainly parameter $\sigma \in[a, b]$ generates a strongly continuous semi-group $(S(t, \sigma))_{t \geqslant 0}$ on $L^{2}(\Omega)$. and $B$ is a control operator in $L\left(\mathbb{R}^{\mathrm{m}}, \mathrm{L}^{2}(\Omega)\right)$ considered to be independent of $\sigma$ and $p \in \mathrm{L}^{2}\left(0, T, \mathbb{R}^{\mathrm{m}}\right)$ is a distributed control which does not depend on $\sigma, L^{2}\left(0, T, \mathbb{R}^{m}\right)$ is a space of controls. $u_{0} \in L^{2}(\Omega)$ are independent of the parameter $\sigma . u_{p}(t, \sigma)$ denote the solution of the first equation given by

$$
u_{p}(t, \sigma)=S(t, \sigma) y_{0}+\int_{0}^{t} s(t-s, \sigma) B p(s) d s,
$$

where we suppose $\omega \subset \Omega$ and $\mathrm{u}_{\mathrm{d}} \in \mathrm{L}^{2}(\omega)$.

Next, we formulate the notion of regional averaged controllability which can be found using the regional controllability definitions introduced by El Jai in [3] averaged controllability proposed by Zuazua in [14].

\section{Definition 2.1.}

1. The system (2.1) is called $\omega$-exactly regionally averaged controllable to $u_{d} \in L^{2}(\omega)$ if there exists a control $p \in \mathrm{L}^{2}\left(0, \mathrm{~T}, \mathbb{R}^{\mathrm{m}}\right)$ independent of the parameter $\sigma$ such that

$$
u_{d}=\frac{1}{b-a} \int_{a}^{b} u_{p}(T, \sigma) d \sigma .
$$

2. The system (2.1) is called $\omega$-approximately regionally averaged controllable for all $\varepsilon>0$ if there exists a control $p \in L^{2}\left(0, T, \mathbb{R}^{m}\right)$ independent of the parameter $\sigma$ such that

$$
\left\|\frac{1}{b-a} \int_{a}^{b} u_{p}(T, \sigma) d \sigma-u_{d}\right\| \leqslant \varepsilon .
$$

Remark 2.2. Clearly the definitions notify that we are only interested in steering the state average (relative to such a parameter) to the desired state over the subregion $\omega$.

Let for each $\sigma \in[a, b]$

$$
\begin{gathered}
H_{\sigma}: L^{2}\left(0, T, \mathbb{R}^{m}\right) \rightarrow L^{2}(\Omega), \\
H_{\sigma} \cdot p=\int_{0}^{T} s(T-s, \sigma) B p(s) d s,
\end{gathered}
$$

and let the restriction function defined by

$$
\begin{aligned}
\chi_{\omega} \quad \mathrm{L}^{2}(\Omega) & \rightarrow \mathrm{L}^{2}(\omega) \\
\mathrm{u} & \left.\mapsto \mathrm{u}\right|_{\omega,}
\end{aligned}
$$


where the adjoint is defined by

$$
\begin{aligned}
\chi_{\omega}^{*} \mathrm{~L}^{2}(\omega) & \rightarrow \mathrm{L}^{2}(\Omega) \\
\mathrm{u} & \mapsto\left\{\begin{array}{l}
u \text { in } \omega \\
0 \text { in } \Omega \backslash \omega,
\end{array}\right.
\end{aligned}
$$

The system (2.1) is $\omega$-exactly regionally averaged controllable if

$$
\operatorname{Im} \chi_{\omega} \frac{1}{b-a} \int_{a}^{b} H_{\sigma} d \sigma=L^{2}(\omega)
$$

The system (2.1) is $\omega$-approximately regionally averaged controllable if

$$
\overline{\operatorname{Im} \chi_{\omega} \frac{1}{b-a} \int_{a}^{b} H_{\sigma} d \sigma}=L^{2}(\omega) .
$$

Remark 2.3.

1. We can remark that the regional average controllability is weaker than the regional controllability, since we just control the average of the state.

2. The definition that we discuss concerned the transfer of the average of the state subject to the unknown parameter on a region $\omega$.

Proposition 2.4. The system (2.1) is w-exactly regionally averaged controllable if and only if

$$
\operatorname{Ker} \chi_{\omega}+\operatorname{Im} \frac{1}{b-a} \int_{a}^{b} H_{\sigma} d \sigma=L^{2}(\Omega) .
$$

The system (2.1) is w-approximately regionally averaged controllable if and only if

$$
\operatorname{Ker\chi } \chi_{\omega}+\overline{\operatorname{Im} \frac{1}{b-a} \int_{a}^{b} H_{\sigma} d \sigma}=L^{2}(\Omega) .
$$

Proof. The proof of this proposition is a simple exercise of Controllability. The equalities (2.6) and (2.7) follows from (2.1) with (2.5). The details are straightforward and therefore omitted here.

\section{Regional averaged controllability problem}

Now, we list averaged control problem with the minimum energy that guarantee the transfer of the average solution to a desired state on $\omega$. The approach developed in this paper is an extension of HUM approach [4]. fHUM approach consists on putting the regional controllability problem as a particular case of the Global controllability problem see [3] for instance.

Let us consider the system (2.1) and $A \in \mathrm{L}^{2}(\Omega)$

$$
\begin{cases}\frac{d u}{d t}=A(\sigma) u+B p & t \in(0, T) \\ u=0 & \partial \Omega \times(0, T) \\ u(0)=u_{0} \in D(A(\sigma)) . & \end{cases}
$$

Note that, using [4], we can proof that (3.1) admit a unique solution $\mathfrak{u}_{\mathfrak{p}} \in \mathrm{L}^{2}(\Omega)$.

Let

$$
\left\{\begin{array}{l}
\min _{\substack{p \in \mathrm{L}^{2}(0, T) \\
\text { such that }}} p^{*}=\|p\|_{\mathrm{L}^{2}(0, T)}^{2}=\frac{1}{b-a} \int_{a}^{b} u_{p}(T, \sigma) d \sigma \text { in } \omega
\end{array}\right.
$$


where $u_{d} \in L^{2}(\omega)$ is a desired target at time T. Let $G$ be a set (closed subspace) of $L^{2}(\Omega)$ given by

$$
\mathrm{G}=\left\{u \in \mathrm{L}^{2}(\Omega) ; u=0 \text { in } \omega\right\}
$$

The problem is to search if there exist a minimum norm of the control $p \in \mathrm{L}^{2}\left(0, T, \mathbb{R}^{\mathrm{m}}\right)$ such that $u-u_{d} \in$ G. Furthermore, we introduce the parameter dependent adjoint system in which the data at the final time $\mathrm{t}=\mathrm{T}, v \in \mathrm{G}^{*}$ are independent of the uncertainty parameter $\sigma$.

$$
\left\{\begin{array}{l}
\frac{\mathrm{d} v}{\mathrm{dt}}=-\mathrm{A}^{*}(\sigma) v \quad \mathrm{t} \in(0, \mathrm{~T}) \\
v(\mathrm{~T})=v^{0}
\end{array}\right.
$$

with

$$
\mathrm{G}^{*}=\left\{v \in \mathrm{L}^{2}(\Omega) ; v=0 \text { in } \Omega \backslash \omega\right\},
$$

and let

$$
\left\|v^{0}\right\|_{G^{*}}^{2}=\frac{1}{b-a} \int_{a}^{b} \int_{0}^{T}\left\|B^{*} v(t)\right\|^{2} d t d \sigma
$$

we also consider

$$
\left\{\begin{array}{l}
\frac{d \varphi}{d t}=-A(\sigma) \varphi+B^{*} \frac{1}{b-a} \int_{a}^{b} v(t) d \sigma, \quad t \in(0, T), \\
\varphi(0)=u_{0}
\end{array}\right.
$$

and define an operator $M$ by

$$
M v^{0}=P\left(\frac{1}{b-a} \int_{a}^{b} \varphi(t, \sigma)\right) d \sigma
$$

where

$$
P=\left.\left.\chi\right|_{\omega} ^{*} \chi\right|_{\omega}
$$

$M$ is affine and can be wrote as

$$
M v^{0}=P\left(\frac{1}{b-a} \int_{a}^{b} \varphi_{0}(t) d \sigma+\frac{1}{b-a} \int_{a}^{b} \varphi_{1}(t) d \sigma\right),
$$

where

$$
\left\{\begin{array}{l}
\frac{d \varphi_{0}}{d t}=A(\sigma) \varphi_{0}(t), \quad t \in(0, T) \\
\varphi_{0}(0)=u_{0}
\end{array}\right.
$$

and

$$
\left\{\begin{array}{l}
\frac{d \varphi_{1}}{d t}=-A(\sigma) \varphi_{1}+B B^{*} \frac{1}{b-a} \int_{a}^{b} v(t) d \sigma, \quad t \in(0, T), \\
\varphi_{1}(0)=0 .
\end{array}\right.
$$

Now, Let the operator $\Theta: G \rightarrow G^{0}$ defined by

$$
\Theta v^{0}=\mathrm{P}\left(\varphi_{1}(\mathrm{t})\right)
$$

Therefore, the regional averaged controllability problem imply to solve the equation

$$
\Theta v^{0}=\chi_{\omega}^{*} u_{d}-P\left(\frac{1}{b-a} \int_{a}^{b} \varphi_{0}(t) d \sigma\right)
$$

\section{Proposition 3.1.}

1. If the system is $\omega$ averaged regionally approximately controllable then (3.4) define a norm.

2. The operator $\Theta$ defined by (3.7) is a positive definite operator. 
Proof.

1. Let $v^{0}$ such that $\left\|v^{0}\right\|_{\mathrm{G}^{*}}^{2}=0$, then $\frac{1}{\mathrm{~b}-\mathrm{a}} \int_{\mathrm{a}}^{\mathrm{b}} \int_{0}^{\mathrm{T}}\left\|\mathrm{B}^{*} v(\mathrm{t})\right\|^{2} \mathrm{dtd} \sigma=0$, from which one can conclude that $\mathrm{B}^{*} v(\mathrm{t})=0$.

While the system (3.1) is $\omega$ averaged approximately regionally controllable, then the kernel of the operator $\mathrm{H}^{*} \chi_{\omega}^{*}$ is zero see [3]. Such kernel can be characterize by $\mathrm{B}^{*} \mathrm{~S}^{*}(T-t, \sigma) v^{0}=0$ which gives $v^{0}=0$ and (3.4) is a norm.

2. By an easy calculus and using (3.7) on can prove that

$$
\left\langle\Theta v^{0}, v^{0}\right\rangle_{\mathrm{G}^{*}, \mathrm{G}}=\left\|v^{0}\right\|_{\mathrm{G}^{*}}^{2}
$$

which show that $\Theta$ is a positive definite operator.

Proposition 3.2. If the system (3.1) is w-approximately averaged regionally controllable then the equation (3.8) has a unique solution $v^{0} \in \mathrm{G}^{0}$ and the control $\mathrm{p}^{*}(\mathrm{t})=\mathrm{B}^{*} \mathrm{v}(\mathrm{t})$ is the minimum of the problem (3.2).

Proof. If we consider (3.1) augmented with the control $p^{*}(t)=B^{*} v(t)$ and using the equations (3.6), (3.7), we conclude that $u_{d}=\frac{1}{b-a} \int_{a}^{b} u_{p}(T, \sigma) d \sigma$ in $\omega$.

For the uniqueness of optimal control, one can choose two optimal controls $p, q$ and using easy calculus prove that derivative of the cost is

$$
\frac{2}{b-a} \int_{a}^{b} \int_{0}^{T}\left(p^{*}, p-q\right) d t d \sigma=0,
$$

and this establishes the optimality of $\mathrm{p}^{*}$.

\section{Gradient average controllability problem}

Let $u_{p}($.$) be the solution to (2.1) excited by a control p$ and suppose that (2.1) has a unique solution such that $u_{p}(T) \in H^{1}(\Omega)$ see [1]. For $\omega \in \Omega$, we consider

$$
\begin{aligned}
\Lambda_{\omega}\left(\mathrm{L}^{2}(\Omega)\right)^{\mathrm{n}} & \rightarrow\left(\mathrm{L}^{2}(\omega)\right)^{\mathrm{n}} \\
\mathrm{u} & \left.\mapsto \mathrm{u}\right|_{\omega,}
\end{aligned}
$$

where the adjoint is defined by

$$
\begin{aligned}
\Lambda_{\omega}^{*}\left(\mathrm{~L}^{2}(\omega)\right)^{\mathrm{n}} & \rightarrow\left(\mathrm{L}^{2}(\Omega)\right)^{\mathrm{n}} \\
\mathrm{u} & \mapsto\left\{\begin{array}{l}
u \text { in } \omega \\
0 \text { in } \Omega \backslash \omega .
\end{array}\right.
\end{aligned}
$$

Let $\nabla$ be the operator given by

$$
\begin{aligned}
\Lambda_{\omega} \quad H^{1}(\Omega) & \rightarrow\left(L^{2}(\Omega)\right)^{n} \\
u & \mapsto \nabla u=\left(\frac{\partial u}{\partial x_{1}}, \cdots, \frac{\partial u}{\partial x_{n}}\right),
\end{aligned}
$$

with adjoint $\nabla^{*}$.

\section{Definition 4.1.}

1. The system (2.1) is said to be $\omega$-exactly regionally gradient averaged controllable to $u_{d}^{g} \in\left(L^{2}(\omega)\right)^{n}$ if there exists a control $p \in \mathrm{L}^{2}\left(0, \mathrm{~T}, \mathbb{R}^{\mathrm{m}}\right)$ independent of the parameter $\sigma$ such that

$$
u_{d}^{g}=\Lambda_{\omega} \nabla\left(\frac{1}{b-a} \int_{a}^{b} u_{p}(T, \sigma) d \sigma\right) .
$$


2. The system (2.1) is said to be $\omega$-approximately regionally averaged controllable for all $\varepsilon>0$ if there exists a control $p \in \mathrm{L}^{2}\left(0, T, \mathbb{R}^{\mathrm{m}}\right)$ independent of the parameter $\sigma$ such that

$$
\left\|\Lambda_{\omega} \nabla\left(\frac{1}{b-a} \int_{a}^{b} u_{p}(T, \sigma) d \sigma\right)-u_{d}^{g}\right\| \leqslant \varepsilon
$$

Let for each $\sigma \in[a, b]$

$$
\begin{gathered}
H_{\sigma}^{g}: L^{2}\left(0, T, \mathbb{R}^{m}\right) \rightarrow H^{1}(\Omega), \\
H_{\sigma}^{g} \cdot p=\int_{0}^{T} s(T-s, \sigma) B p(s) d s .
\end{gathered}
$$

Then the system (2.1) is $\omega$-exactly ( $\omega$-approximately) regionally averaged gradient controllable if and only if

$$
\left.\operatorname{Im} \Lambda_{\omega} \nabla\left(\frac{1}{b-a} \int_{a}^{b} H_{\sigma}^{g} d \sigma\right)=\left(L^{2}(\omega)\right)^{n} \text { resp. } \overline{\left(\operatorname { I m } \Lambda _ { \omega } \nabla \left(\frac{1}{b-a} \int_{a}^{b} H_{\sigma}^{g} d \sigma\right.\right.}\right)=\left(L^{2}(\omega)\right)^{n} .
$$

Now, we are apple to state the next proposition

Proposition 4.2. The system (2.1) is w-exactly regionally averaged gradient controllable if and only if

$$
\operatorname{Ker} \Lambda_{\omega}+\operatorname{Im} \Lambda_{\omega}^{*} \Lambda_{\omega} \nabla\left(\frac{1}{b-a} \int_{a}^{b} H_{\sigma}^{g} d \sigma\right)=\left(L^{2}(\Omega)\right)^{n}
$$

The system (2.1) is w-approximately regionally averaged gradient controllable if and only if

$$
\operatorname{Ker} \chi_{\omega}+\overline{\operatorname{Im} \Lambda_{\omega}^{*} \Lambda_{\omega} \nabla\left(\frac{1}{b-a} \int_{a}^{b} H_{\sigma}^{g} d \sigma\right)}=\left(L^{2}(\Omega)\right)^{n} .
$$

Proof. The proof of this proposition is a simple exercise of Controllability. The equalities (4.3) and (4.4) follows from (2.1) with (2.5). The details are straightforward and therefore omitted here.

So, to explore an approach devoted to the computation of an optimal control for system (2.1) to a given gradient in the subregion $\omega$. Given $u^{g} \in\left(L^{2}(\omega)\right)^{n}$, we set

$$
\begin{cases}\frac{d u}{d t}=A(\sigma) u+\Lambda_{D} f p & t \in(0, T) \\ u=0 & \partial \Omega \times(0, T), \\ u(0)=u_{0} \in D(A(\sigma)) . & \end{cases}
$$

Note that, using [4], we can proof that (4.5) admit a unique solution $u_{p} \in \mathrm{L}^{2}(\Omega)$. Let

$$
\left\{\begin{array}{l}
\min _{p \in \mathrm{L}^{2}(0, T)} p^{*}=\|p\|_{\mathrm{L}^{2}(0, T)}^{2}, \\
\text { such that } u_{d}=\nabla\left(\frac{1}{b-a} \int_{a}^{b} u_{p}(T, \sigma) d \sigma\right) \text { in } \omega,
\end{array}\right.
$$

where $u_{d} \in L^{2}(\omega)$ is a desired target at time T. Let $G$ be a set (closed subspace) of $L^{2}(\Omega)$ given by

$$
\mathrm{G}=\left\{u \in \mathrm{L}^{2}(\Omega) ; u=0 \text { in } \omega\right\} .
$$

The problem is to search if there exist a minimum norm control $p \in L^{2}\left(0, T, \mathbb{R}^{m}\right)$ such that $u_{p}-u_{d} \in G$. Furthermore, we introduce the parameter dependent adjoint system in which the data at the final time $\mathrm{t}=\mathrm{T}, v \in \mathrm{G}^{*}$ are independent of the uncertainty parameter $\sigma$. 
Let the system for $v^{0} \in \mathrm{G} *$

$$
\left\{\begin{array}{l}
\frac{d v}{d t}=-A^{*}(\sigma) v \quad t \in(0, T), \\
v(T)=v^{0}
\end{array}\right.
$$

We assume that it has a unique solution $v \in \mathrm{L}^{2}\left(0, \mathrm{~T} ; \mathrm{H}_{0}^{2}(\Omega)\right) \cap \mathrm{C}^{3}(\Omega \times(0, \mathrm{~T}))$. For a given $v^{0} \in \mathrm{G} *$, we consider system (4.7) and define the mapping

$$
v^{0} \in \mathrm{G} * \rightarrow\left\|v^{0}\right\|_{\mathrm{G} *}^{2}=\frac{1}{\mathrm{~b}-\mathrm{a}} \int_{\mathrm{a}}^{\mathrm{b}} \int_{0}^{\mathrm{T}}\left(\sum_{i=1}^{\mathrm{n}}<\mathrm{f}, \frac{\partial v}{\partial x_{\mathrm{i}}}>_{\mathrm{L}^{2}(\mathrm{D})}\right)^{2} \mathrm{dtd} \sigma,
$$

which is a semi-norm on $\mathrm{G} *$. We denote the completion of the set $\mathrm{G} *$ with respect to the norm (4.8) again by $\mathrm{G} *$ and consider the system

$$
\begin{cases}\left.\frac{d u}{d t}=A(\sigma) u+\Lambda_{D} f \sum_{i=1}^{n}<f, \frac{\partial v}{\partial x_{i}}>_{L^{2}(D)}\right)^{2} & t \in(0, T), \\ u=0 & \partial \Omega \times(0, T), \\ u(0)=u_{0} \in \Omega, & \end{cases}
$$

which may be decomposed in the following two systems

$$
\begin{cases}\frac{d u}{d t}=A(\sigma) u_{1}, & t \in(0, T) \\ u=0, & \partial \Omega \times(0, T) \\ u(0)=u_{0} \in \Omega, & \end{cases}
$$

and

$$
\begin{cases}\left.\frac{d u}{d t}=A(\sigma) u_{2}(x, t, \sigma)+\Lambda_{D} f \sum_{i=1}^{n}<f, \frac{\partial v}{\partial x_{i}}>_{L^{2}(D)}\right)^{2}, & t \in(0, T), \\ u=0, & \partial \Omega \times(0, T), \\ u(0)=u_{0} \in \Omega . & \end{cases}
$$

Let $\wedge$ be the operator defined by

$$
\begin{aligned}
\wedge: \mathrm{G} * & \rightarrow \overline{\mathrm{G} *} \\
v_{0} & \mapsto \frac{1}{\mathrm{~b}-\mathrm{a}} \int_{\mathrm{a}}^{\mathrm{b}} \mathrm{P}\left(\sum_{i=1}^{\mathrm{n}} \frac{\partial u_{2}(x, \mathrm{t}, \sigma)}{\partial x_{i}}(\mathrm{~T})\right) \mathrm{d} \sigma,
\end{aligned}
$$

where $P=\Lambda_{\omega}^{*} \Lambda_{\omega}$. With this notation the regional average gradient control on $\omega$ leads to solving the equation

$$
\bigwedge v_{0}=-\frac{1}{b-a} \int_{a}^{b} P\left(\Lambda_{\omega}\left(g_{d}\right)_{i}-\sum_{i=1}^{n} \frac{\partial u_{1}(x, t, \sigma)}{\partial x_{i}}(T)\right) d \sigma .
$$

Proposition 4.3. If the system (4.5) is w-approximately averaged gradient regionally controllable then the equation (4.13) has a unique solution $v^{0} \in \mathrm{G}^{0}$ and the control $\mathrm{p}^{*}(\mathrm{t})=\langle\mathrm{F}, \nabla v(\mathrm{t})>$ is the minimum of the problem (4.6).

Proof. By the same way as the proof of proposition (3.1), one can prove that (4.8) is a norm and that (4.12) is a well definite positive operator. If we consider (4.5) augmented with the control $\mathrm{p}^{*}(\mathrm{t})=\langle\mathrm{F}, \nabla v(\mathrm{t})\rangle$ and using the equations (4.7), (4.12), we conclude that $u_{d}=\nabla\left(\frac{1}{b-a} \int_{a}^{b} u_{p}(T, \sigma) d \sigma\right)$ in $\omega$.

For the uniqueness of optimal control, one can choose two optimal controls p, q and using easy calculus prove that derivative of the cost is

$$
\frac{2}{b-a} \int_{a}^{b} \int_{0}^{T}\left(p^{*}, p-q\right) d t d \sigma=0,
$$

and this establishes the optimality of $\mathrm{p}^{*}$. 


\section{Conclusion}

Regional controllability treats many problems using independent parameter systems. In this document, we introduce the notion of regional averaged controllability which allows us to consider optimal control problems using dependent parameter systems. We give the definitions and establish the necessary conditions for the regional averaged controllability and the regional averaged gradient controllability of linear systems. We define the averaged optimal control problem and the averaged gradient optimal control problem with minimum energy. We propose the solutions of such problems using an extension of HUM. The paper opens a wide way of research studying other types of semi-linear or nonlinear systems. The proposed method can be generalized to the case of hyperbolic distributed systems, the average controllability of hyperbolic systems can be considered as in [11-13].

\section{References}

[1] H. Brezis, Analyse fonctionnelle, Masson, Paris, (1983). 4

[2] A. El Jai, A. J. Pritchard, Capteurs et actionneurs dans l'analyse des systeemes distribuees, Research in Applied Mathematics, Masson, Paris, (1986). 1

[3] A. El Jai, M. C. Simon, E. Zerrik, A. J. Pritchard, Regional controllability of distributed parameter systems, Internat. J. Control, 62 (1995), 1351-1365. 2, 3, 1

[4] J.-L. Lions, E. Magenes, Problémes aux limites non homogénes et applications, Dunod, Paris, (1968). 1, 3, 3, 4

[5] J. Lohéac, E. Zuazua, Averaged controllability of parameter dependent conservative semigroups, J. Differential Equations, 262 (2017), 1540-1574. 1

[6] Q. Lü, E. Zuazua, Averaged controllability for random evolution partial differential equations, J. Math. Pures Appl. (9), 105 (2016), 367-414. 1

[7] M. Ould Sidi, S. A. Beinane, Regional gradient optimal control problem governed by a distributed bilinear systems, Telkomnika, 17 (2019), 1957-1965. 1

[8] E. Zerrik, A. Boutoulout, A. Kamal, Regional Controllability of Parabolic systems, Int. J. Appl. Math. Comput. Sci., 9 (1999), 767-787. 1

[9] E. Zerrik, A. Kamal, A. Boutoulout, Regional gradient controllability and actuators, Internat. J. Systems Sci., 33 (2002), 239-246. 1

[10] E. Zerrik, A. Kamal, A. Boutoulout, Regional flux target with minimum energy, Control Theor. Appl., 149 (2002), 349-356. 1

[11] R. Zine, Optimal control for a class of bilinear hyperbolic distributed systems, Far East J. Math. Sci., 102 (2017), 17611775. 5

[12] R. Zine, M. Ould Sidi, Regional optimal control problem with minimum energy for a class of bilinear distributed systems, IMA J. Math. Control Inform., 35 (2018), 1187-1199.

[13] R. Zine, M. Ould Sidi, Regional optimal control problem governed by distributed bilinear hyperbolic systems, Int. J. Control Autom. Syst., 16 (2018), 1060-1069. 5

[14] E. Zuazua, Averaged control, Automatica J. IFAC, 50 (2014), 3077-3087. 1, 2 